\title{
Estimación de los Factores Situacionales del Proceso de Requisitos
}

\author{
Viviana A. Ledesma ${ }^{1,2}$, Graciela D. Hadad ${ }^{1,2}$, Jorge H. Doorn ${ }^{1,2}$ y Nicolás A. Bedetti ${ }^{2}$ \\ ${ }^{1}$ Escuela de Informática, Universidad Nacional del Oeste, Argentina \\ ${ }^{2}$ DIIT, Universidad Nacional de La Matanza, Argentina \\ vledesma@unlam.edu.ar; ghadad@uno.edu.ar; \\ jdoorn@exa.unicen.edu.ar; nbedetti@unlam.edu.ar
}

\begin{abstract}
Resumen. Para obtener un producto software de buena calidad es vital aplicar un proceso apropiado de requisitos. En ese sentido, la literatura ofrece procesos de requisitos bien definidos de muy diverso alcance. Sin embargo, la experiencia indica que gran parte de los proyectos reales tienen características que hacen conveniente efectuar cambios en los procesos. Para ello, es necesario disponer de un método claro que guíe cómo adaptar el proceso en cada situación. Estas situaciones se pueden caracterizar a través de un conjunto de factores observables. Usualmente es difícil establecer con precisión el valor de estos factores al inicio del proceso de requisitos. También debe considerarse la posible evolución de ciertos factores por cambios en el contexto. En otras palabras, a veces no alcanza con solo adaptar un proceso antes de su puesta en ejecución, sino que este puede requerir readaptaciones durante su desarrollo, ya sea por precisarse factores con valoraciones iniciales dudosas o incorrectas, o por ocurrir cambios en el contexto que implican cambios en algunos factores. Finalmente, es necesario estimar cuan viable resulta establecer los factores con una confiabilidad tal que su aplicación para adaptar el proceso resulte aceptable. Por tal motivo, se ha estudiado la evolución de 21 factores durante la aplicación de un proceso de requisitos en 35 proyectos de la industria. Esto ha permitido corroborar la evolución de las situaciones en las que está inmerso un proceso de requisitos y, asimismo, identificar factores a los cuales debe prestarse mayor atención para una adecuada adaptación dinámica del proceso.
\end{abstract}

Palabras Clave: Proceso de Requisitos, Factores Situacionales, Variabilidad de Procesos, Ingeniería de Métodos Situacional.

\section{$1 \quad$ Introducción}

La literatura muestra que las técnicas de elicitación de conocimiento utilizadas en el proceso de Ingeniería de Requisitos (IR) se eligen a menudo en función de las características particulares del contexto de aplicación, principalmente para aquellas fuentes de información que involucran seres humanos [1]. Sin embargo, los factores situacionales que caracterizan esas particularidades, rara vez se toman en cuenta en otras actividades del proceso de requisitos. Sería conveniente que las peculiaridades, que 
podrían obstaculizar o favorecer un proceso de IR, sean identificadas al comienzo del proyecto a fin de permitir su adaptación. Sin embargo, algunos factores pueden evolucionar a lo largo del ciclo de desarrollo del software lo que hace necesaria la readaptación del proceso en posteriores hitos estratégicos.

El objetivo del presente trabajo es estudiar la percepción de los ingenieros de requisitos respecto de la situación imperante en el proceso de IR, a través de la valoración del conjunto de factores que caracterizan dicha situación y la verosimilitud o estabilidad de dichas valoraciones a lo largo del proceso. Debe para ello tenerse en cuenta que si bien las evoluciones a lo largo del proyecto son generalmente impredecibles, las apreciaciones iniciales dudosas o incorrectas son potencialmente reducibles. Es entonces relevante la calidad con que es posible realizar la estimación de los factores situacionales, y en consecuencia la calidad de la adaptación del proceso de requisitos a su contexto.

En la sección siguiente se exponen conceptos sobre la variabilidad de procesos y enfoques cuyo conocimiento es necesario para crear o adaptar procesos de software, y específicamente procesos de requisitos, en función de los factores que caracterizan esa variabilidad. En la sección 3 se describe un conjunto de factores situacionales, sus interacciones y su tipo de evolución. En la sección 4 se presenta el estudio de caso llevado a cabo para ponderar la calidad de estimación de los factores situacionales y en la sección 5 se analizan los resultados obtenidos. Finalmente, se exponen conclusiones y futuros trabajos.

\section{$2 \quad$ Adaptabilidad de Procesos}

Uno de los desafíos que enfrentan las organizaciones tiene que ver con optimizar la gestión de sus procesos en entornos altamente dinámicos, por lo que disponer de procesos que sean flexibles y puedan ajustarse para soportar posibles cambios en ese entorno, resulta clave para su éxito [2]. La gestión de la variabilidad de procesos permite hacer frente a esta problemática, siendo aplicable en distintos áreas [3, 4, 5].

La variabilidad es la habilidad de un proceso o un producto de ser extendido, personalizado o configurado para ser utilizado bajo un determinado contexto [6]. Por ende, la variabilidad implica proyectar o adelantarse a los cambios, en vez de improvisar al surgir una necesidad o situación no prevista [7]. La gestión de la variabilidad involucra definir:

- los puntos de variación en el proceso, que corresponden a los momentos u oportunidades donde los elementos del proceso pueden cambiar;

- los posibles flujos variantes, que son las partes del proceso que representan la variabilidad y que serán implementados en los puntos de variación; y

- el mecanismo de variabilidad a usar, que puede ser: parametrización, adición, omisión, reemplazo de un elemento simple y variabilidad de tipo de dato.

Desde otra perspectiva, la Ingeniería de Métodos Situacional (IMS) tiene por objetivo construir métodos específicos que se ajusten a las situaciones particulares de su contexto, de modo flexible, adaptable, ágil y repetible; enfocándose en el diseño, construcción y adaptación de métodos, técnicas y herramientas de soporte para el desarrollo de sistemas de software $[8,9,10]$. 
Bajo esta disciplina, se entiende como situación a la combinación de circunstancias en un momento dado y en un contexto específico, que afecta la forma de trabajo y los productos a generar. Una situación debe poder describirse a partir de un conjunto de factores, que sirven de base para determinar la adaptación a realizar sobre el proceso de software [11]. Estos factores muestran particularidades del contexto de aplicación y de las características propias del proyecto de software [10].

Entonces, la forma más eficaz de adaptar un proceso consiste en fragmentar el mismo en bloques más pequeños, los que deben tener un objetivo propio, cumplible mediante una o más actividades, las que se realizan aplicando una o más técnicas incluidas en ese bloque y que pueden generar uno o más artefactos [8]. Algunos bloques son comunes a todas las situaciones, mientras que otros dependen de ciertos factores de situación. Por lo tanto, el proceso se conforma con la composición de los bloques apropiados para una situación particular [12].

Contrastando el enfoque de la IMS con el de variabilidad de procesos, se observa que ambos favorecen la adaptabilidad en base a características particulares y la reutilización. Sin embargo, la IMS presenta una visión más general, dirigida a la construcción de un proceso para una situación determinada a partir de bloques que existen o pueden ser creados, en cambio, la variabilidad parte de un proceso base ya existente y se avoca a establecer sus variaciones posibles o caminos alternativos en función de condiciones pre-definidas que pueden darse al aplicar dicho proceso.

En lo que respecta a la Ingeniería de Requisitos, se han generado varias propuestas orientadas a la creación y mejora de sus procesos, acorde a particularidades propias de cada proyecto. Estos trabajos se pueden catalogar en:

A) Orientados a definir un proceso de IR utilizando frameworks. Se pone a disposición una base de conocimiento sobre actividades y técnicas de IR, que sirve de guía para obtener un proceso de IR acorde a las necesidades del proyecto [13, 14].

B) Enfoques para seleccionar técnicas de elicitación que mejor se adaptan al contexto situacional. Estos trabajos solo contemplan la actividad de elicitación en el proceso de IR $[1,15,16]$.

C) Modelos que permiten adaptar el proceso de IR para una clase específica de proyecto. Se trata de propuestas para la adaptación situacional para proyectos de determinada naturaleza o alcance, por ejemplo, desarrollo global de software o métodos ágiles [17, 18].

D) Guías que facilitan la creación de un proceso de IR adaptado al contexto de aplicación. Aportan mecanismos para la creación de un proceso de IR ajustado a las características situacionales del entorno que, a diferencia del grupo $\mathrm{C}$, pueden aplicarse en proyectos de diferente naturaleza [19, 20].

En todos estos trabajos, se aplican en mayor o menor medida algunas nociones de la IMS, y se proponen algunos factores situacionales que son evaluados con el objetivo de definir el contexto. Particularmente, en los grupos A y D se han encontrado enfoques basados en el uso de distintos componentes para conformar el nuevo proceso de IR. Por su parte, solo en el grupo D existen propuestas que combinan los conceptos de la IMS con algunos principios de variabilidad de procesos.

El trabajo presentado por [15] (grupo B) plantea un enfoque parcialmente dinámico, destacando que el conocimiento acerca del domino del problema y del dominio del proyecto se va adquiriendo a medida que avanza el proceso de IR, con lo cual en 
cada iteración se pueden seleccionar técnicas de elicitación más apropiadas. Además, mencionan que las características situacionales del contexto del problema suelen ser estáticas mientras que las relacionadas con el proyecto pueden cambiar, aunque no presentan evidencia al respecto. En trabajos posteriores donde presentan una ontología con cerca de 50 características situacionales [16], no abordan el tema de la evolución de dichos factores, ni de la calidad con que estiman esos factores. En ese sentido, Rolland [9] también ha señalado la existencia de factores contingentes que cambian durante el ciclo de vida del proyecto, lo que debería acarrear una adaptación dinámica de los procesos.

En la gran mayoría de los estudios relacionados con la IMS no se abordan dos aspectos esenciales relacionados con: la adaptación incorrecta del proceso, y la probabilidad de evolución o de estimación incorrecta de los factores que impulsan esa adaptación. La primera cuestión puede ocurrir bajo 3 variantes: i) la omisión inapropiada de hacer una actividad que hubiera sido necesaria, ii) el reemplazo incorrecto de una actividad por otra, o iii) la inserción innecesaria de una actividad. Es claro que el último este aspecto es fuertemente dependiente del segundo, por lo que un estudio de la evolución y estimación de los factores situacionales puede revelar cuestiones no tan fácilmente visibles, que terminan en adaptaciones fallidas.

El trabajo de investigación en curso, cuya parte referida a la evolución y estimación de factores se presenta en este artículo, se enmarca en el grupo D, pues tiene por finalidad establecer mecanismos claros para adaptar un proceso de IR de acuerdo a las características particulares del contexto de aplicación y del proyecto, y ajustarlo en función de esa evolución, considerando que la misma puede provenir no solo de un cambio real en la situación sino también de una mejor comprensión de esa situación. Para ello, el proceso de adaptación propuesto realiza reevaluaciones de los factores en ciertos hitos del proceso de IR, establecidos como puntos de variación, guiando un reajuste del proceso de IR [21].

\section{Factores Situacionales en un Proceso de Requisitos}

Con base en la propuesta de la IMS, se han estudiado diversos factores considerados en la literatura [1, 20] para distintas actividades de la IR. Como resultado de ese estudio [22] y de la experiencia adquirida a lo largo de más de dos décadas en un proceso de IR basado en modelos en lenguaje natural [23], se identificaron 21 factores situacionales (ver Tabla 1). Debido a que es frecuente que las condiciones iniciales de un proyecto e incluso el contexto de aplicación sean mal percibidas o se alteren durante el proceso, es necesario prestar atención al tipo de evolución que dichos factores sufren. Desde esta dimensión pueden clasificarse en (ver tercera columna en Tabla 1):

- Invariante: aquel factor que, por su propia naturaleza, no tiene cambios durante el proceso de IR.

- Contingente: aquel factor que puede evolucionar naturalmente debido a cambios en el contexto de aplicación y/o en el contexto del proyecto.

Otro aspecto a considerar es el nivel de confianza de los ingenieros de requisitos respecto al valor asignado a cada factor situacional. Desde esta perspectiva, los valores de los factores se tipifican en: 
- Seguro, cuando se considera un nivel alto de certeza al valor asignado al factor, lo que indicaría en principio que sólo podrían ocurrir cambios en aquellos catalogados como contingentes y sólo debido a cuestiones relacionadas con el contexto.

- Razonablemente seguro, cuando se considera que es baja la probabilidad de haberse cometido un error en su estimación.

- Dudoso, cuando no es posible establecer el valor del factor con precisión, sino recién cuando se logre una mejor comprensión de la situación.

Tabla 1. Factores Situacionales bajo estudio

\begin{tabular}{|l|r|c|c|}
\hline \multicolumn{1}{|c}{ Factor Situacional del Contexto de Aplicación } & \multicolumn{2}{c|}{ Valores Admisibles } & Evolución \\
\hline Complejidad del Contexto & Muy Alta/Alta/Media/Baja/Muy Baja & Invariante \\
\hline Tipo de Cliente & Cliente Específico/Mercado Potencial & Invariante \\
\hline Novedad del Contexto & Muy Alta/Alta/Media/Baja/Muy Baja & Contingente \\
\hline Reingeniería en el Proceso de Negocio & Muy Alta/Alta/Media/Baja/Muy Baja & Invariante \\
\hline Volatilidad del Contexto & Muy Alta/Alta/Media/Baja/Muy Baja & Contingente \\
\hline Volatilidad de Requerimientos del Cliente & Muy Alta/Alta/Media/Baja/Muy Baja & Invariante \\
\hline Inconsistencias en el Contexto & Muy Alta/Alta/Media/Baja/Muy Baja & Contingente \\
\hline Conflicto de Intereses de Usuarios & Muy Alta/Alta/Media/Baja/Muy Baja & Invariante \\
\hline Rotación de Usuarios & Valores Admisibles & Evolución \\
\hline \multicolumn{1}{|c|}{ Factor Situacional del Proyecto de Software } & Muy Alta/Alta/Media/Baja/Muy Baja & Contingente \\
\hline Conocimiento Previo del Dominio & Muy Alta/Alta/Media/Baja/Muy Baja & Contingente \\
\hline Envergadura del Proyecto & Muy Alta/Alta/Media/Baja/Muy Baja & Invariante \\
\hline Rotación del Equipo Desarrollador & Muy Alta/Alta/Media/Baja/Muy Baja & Invariante \\
\hline Calidad Exigida del Software & Sí/No & Invariante \\
\hline Reuso de Artefactos de Requisitos Existentes & Sí/No & Invariante \\
\hline Creación de Artefactos de Requisitos para Reuso & Sí/No & Invariante \\
\hline Pre-Rastreabilidad de Requisitos & Sí/No & Invariante \\
\hline Post-Rastreabilidad de Requisitos & Individual/Grupal & Invariante \\
\hline Granularidad de Rastreabilidad de Requisitos & Sí/No & Invariante \\
\hline Exigencia de Producir el Documento ERS & Cascada/Incremental/Iterativo/Ágil & Invariante \\
\hline Restricciones de Tiempo y Recursos del Proyecto & Muy Alta/Alta/Media/Baja/Muy Baja & Contingente \\
\hline Modelo de Proceso de Software & &
\end{tabular}

Entonces, se debe evaluar cada factor y estimar su valor (ver Tabla 1), otorgándole un nivel de confianza. Esto es necesario ya que, en algunos casos, los factores pueden no conocerse de manera precisa. Aquellos factores cuyo nivel de confianza es Dudoso requieren ser observados a medida que el proceso avanza, con el objeto de establecer su valor con mayor precisión y, de ser necesario, re-adaptar el proceso de IR.

Por otra parte, algunos factores tienen influencia sobre otros. Si se considera que A y B son distintos factores situacionales, puede existir alguna de estas interacciones:

- Interacción neutra: indica que los factores A y B son totalmente independientes entre sí, no existe influencia entre ambos.

- Interacción invalidante: indica que si el factor A toma cierto valor no se debe tener en cuenta el factor B. Ejemplo: Si Novedad del Contexto es "Sí", no se consideran los factores Reingeniería en el Proceso de Negocio, Inconsistencias en el Contexto, Conflicto de Intereses de Usuarios y Rotación de Usuarios.

- Interacción limitante: señala que si el factor A toma cierto valor entonces se restringen los valores que puede tomar el factor B. Ejemplo: Si Volatilidad del contexto es "Alta" o "Muy Alta", Modelo de Proceso de Software no puede tomar el valor "Cascada". 
- Interacción incompatible: indica que A y B no pueden tomar a la vez ciertos valores, por lo que se requiere corregir la incompatibilidad mediante acuerdo entre los involucrados para modificar el valor de uno o todos los factores afectados. Ejemplo: Se debe negociar si Restricciones de Tiempo y Recursos para el Proyecto es "Alto" o "Muy Alto" y Calidad Exigida del Software es "Alta" o "Muy Alta".

\section{Descripción del Estudio de Caso sobre Factores Situacionales}

Son muy difundidos los peligros del estudio de caso como método para la comprensión de un problema [24] en virtud de sus características de ser intuitivo, primitivo y difícil de gestionar. Aunque parte de estos peligros no son tales, sino que provienen de la usual confusión entre los tipos de evidencias manipuladas (datos cualitativos o cuantitativos), los mecanismos de recolección de información y la estrategia de investigación (estudio de caso). Claramente, el estudio de caso puede combinarse con cualquier forma de evidencia y con cualquier mecanismo de recolección de información [25]. La diferencia entre un estudio de un caso y un experimento es que en el primero se examina un fenómeno en su contexto real, especialmente cuando dicho fenómeno no puede separarse fácilmente de su contexto o cuando esta interrelación es de interés, mientras que en el segundo se separa deliberadamente el fenómeno de su contexto.

La presencia combinada del fenómeno y su contexto genera la presencia de tantas variables que hace inviable el diseño de experimentos clásicos. Es por ello que en el presente trabajo se llevó a cabo el estudio de caso.

Este consistió en evaluar los factores situacionales usados para definir cómo ajustar el proceso de IR en una situación específica, en el contexto de personas que están adquiriendo conocimiento de tres dominios en forma concurrente; estos dominios son: i) el proceso de requisitos, ii) la estimación de los factores situacionales, y iii) un proceso de negocios de la vida real en el que se planifica incorporar un sistema de software. Este caso, aun con la gran imprecisión en la definición de sus límites, permite estimar la viabilidad de la aplicación de la IMS en problemas de la vida real y obtener información acerca de la validez de las suposiciones realizadas en forma preliminar acerca de la variabilidad de los factores situacionales a lo largo del proceso de IR.

La cantidad de factores situacionales, 21, y la cantidad de clases cualitativas posibles (ver Tabla 1) hacen que la cantidad de combinaciones, 1680, sea imposible de cubrir, aun con un ejemplar por combinación. Además, como los procesos del negocio utilizados fueron tomados estrictamente de la realidad, no resulta siquiera posible tener un ejemplar para cada posible valor de cada uno de los factores situacionales. Esto se muestra en la Tabla 2 en la que el factor situacional Complejidad del Contexto no tiene un solo ejemplar para los valores "Muy Alta" y "Muy Baja". Pese a todas las limitaciones indicadas, el presente estudio trasciende la característica de exploratorio, siendo en todos sus aspectos, descriptivo y en mucho de ellos explicativo.

Entonces, se realizó el estudio sobre 35 proyectos para evaluar la evolución de los factores situacionales y el nivel de confianza asignado a cada valor en contextos de organizaciones reales para la producción de requisitos de software.

El estudio se llevó a cabo siguiendo el mismo proceso de requisitos [23] en todos 
los proyectos, sin ser adaptado a la situación concreta de cada proyecto. Los proyectos se originaron en diferentes organizaciones del Área Metropolitana de Buenos Aires. Cada proyecto fue desarrollado por un equipo diferente, conformado por 4 a 6 alumnos de $4^{\circ}$ año de la carrera de Ingeniería en Informática. Cada proyecto se completó durante un cuatrimestre (16 semanas) del curso de Ingeniería de Requisitos, entre 2015 y 2017. Los proyectos se desarrollaron estudiando el contexto de aplicación en cada organización y planteando una solución a través de un Documento de Especificación de Requisitos bajo el estándar IEEE Std 29148:2011. Como parte del proceso se construyeron tres tipos de modelos en lenguaje natural [23], aplicando técnicas de elicitación tradicionales, negociación, inspecciones, validaciones informales, versionado de modelos y rastreabilidad mediante matrices.

Tabla 2. Datos recolectados de tres Factores Situacionales

\begin{tabular}{|c|c|c|c|c|c|c|c|c|c|c|c|c|c|c|}
\hline \multirow{3}{*}{$\begin{array}{l}\text { Pro- } \\
\text { vecto }\end{array}$} & \multirow{3}{*}{$\begin{array}{l}\text { CUATRl- } \\
\text { MESTRE }\end{array}$} & \multirow[b]{3}{*}{ AÑo } & \multicolumn{4}{|c|}{ Complejidad del contexto } & \multicolumn{4}{|c|}{ Volatilidad del contexto } & \multicolumn{4}{|c|}{ Volatilidad de los requerimientos del cliente } \\
\hline & & & \multicolumn{2}{|c|}{ VALORES } & \multicolumn{2}{|c|}{ CONFIANZA } & \multicolumn{2}{|c|}{ VALORES } & \multicolumn{2}{|c|}{ CONFIANZA } & \multicolumn{2}{|c|}{ VALORES } & \multicolumn{2}{|c|}{ CONFIANZA } \\
\hline & & & Inicial & Final & Inicial & Final & Inicial & Final & Inicial & Final & Inicial & Final & Inicial & Final \\
\hline 1 & & 2015 & Baja & Baja & Seguro & Seguro & Muy Baja & Muy Baja & Seguro & Seguro & Baja & Muy Baja & Seguro & Seguro \\
\hline$\frac{2}{2}$ & 2 & 2015 & Media & Baja & Seguro & Seguro & Baja & Baja & Seguro & Rz Seguro & Baja & Baja & Seguro & Dudoso \\
\hline 3 & 2 & 2015 & Media & Media & Seguro & Seguro & Baja & Baja & Seguro & Seguro & Baja & Media & Seguro & Rz Seguro \\
\hline 4 & 2 & 2015 & Baja & Baja & Seguro & Seguro & Baja & Muy Baja & Rz Seguro & Seguro & Baja & Baja & Rz Seguro & Seguro \\
\hline 5 & 2 & 2015 & Media & Media & Rz Seguro & Seguro & Media & Muy Alta & Rz Seguro & Rz Seguro & Alta & Muy Alta & Rz Seguro & Seguro \\
\hline 6 & 2 & 2015 & Media & Media & Rz Seguro & Seguro & Baja & Baja & Rz Seguro & Seguro & Baja & Baja & Rz Seguro & Seguro \\
\hline 7 & 2 & 2015 & Baja & Media & Rz Seguro & Rz Seguro & Baja & Baja & Dudoso & Rz Seguro & Baja & Baja & Rz Seguro & Rz Seguro \\
\hline 8 & 2 & 2015 & Baja & Baja & Seguro & Rz Seguro & Baja & Muy Baja & Seguro & Seguro & Media & Muy Baja & Rz Seguro & Rz Seguro \\
\hline 9 & 2 & 2015 & Baja & Baja & Rz Seguro & Seguro & Media & & Rz Segure & & Media & Media & Dudoso & Seguro \\
\hline 10 & 2 & 2015 & Media & Baja & Seguro & Seguro & Muy Baja & Baja & Seguro & Seguro & Muy Baja & Baja & Seguro & Seguro \\
\hline 11 & 2 & 2015 & Baja & Baja & Rz Seguro & Seguro & Baja & Muy Baja & Seguro & Seguro & Media & Baja & Rz Seguro & Seguro \\
\hline 12 & 2 & 2015 & Media & Media & Seguro & Seguro & Alta & Media & Rz Seguro & Rz Seguro & & Baja & & Rz Segure \\
\hline 13 & 1 & 2016 & Media & Baja & Rz Seguro & Seguro & Baja & Alta & Seguro & Seguro & Media & Alta & Seguro & Seguro \\
\hline 14 & 1 & 2016 & Alta & Media & Seguro & Seguro & Baja & Baja & Rz Seguro & Seguro & Baja & Baja & Seguro & Seguro \\
\hline 15 & 1 & 2016 & Alta & Media & Seguro & Seguro & Alta & Alta & Seguro & Rz Seguro & Alta & Baja & Rz Seguro & Seguro \\
\hline 16 & 1 & 2016 & Media & Media & Rz Seguro & Seguro & Baja & Baja & Rz Seguro & Seguro & Baja & Media & Rz Seguro & Seguro \\
\hline 17 & 1 & 2016 & Media & Media & Dudoso & Seguro & Muy Baja & Baja & Seguro & Seguro & Muy Baja & Baja & Seguro & Seguro \\
\hline 18 & 2 & 2016 & Media & Media & Rz Seguro & $\begin{array}{ll}\mathrm{Rz} \text { Seguro } \\
\end{array}$ & Baja & Baja & Seguro & Rz Seguro & Media & Media & Seguro & Dudoso \\
\hline 19 & 2 & 2016 & Media & Media & Rz Seguro & Seguro & Media & Baja & Rz Seguro & Rz Seguro & Alta & Baja & Rz Seguro & Seguro \\
\hline 20 & 2 & 2016 & Media & Media & Seguro & Seguro & Muy Baja & Baja & Seguro & Rz Seguro & Baja & Baja & Rz Seguro & Seguro \\
\hline 21 & 2 & 2016 & Media & Media & Rz Seguro & Seguro & Muy Baja & Baja & Seguro & Seguro & Baja & Baja & Seguro & Seguro \\
\hline 22 & 2 & 2016 & Media & Baja & Rz Seguro & Seguro & Baja & Muy Baja & Seguro & Seguro & Baja & Baja & Seguro & Seguro \\
\hline 23 & 2 & 2016 & Media & Media & Seguro & \begin{tabular}{|l}
$\mathrm{Rz}$ Seguro \\
\end{tabular} & Baja & Baja & Rz Seguro & Seguro & Baja & Baja & Seguro & Seguro \\
\hline 24 & 2 & 2016 & Baja & Media & Rz Seguro & Seguro & Baja & Muy Baja & Seguro & Seguro & Baja & Baja & & Rz Segure \\
\hline 25 & 1 & 2017 & Media & Baja & Rz Seguro & Seguro & Muy Baja & Baja & Seguro & Seguro & Muy Baja & Baja & Seguro & Seguro \\
\hline 26 & 1 & 2017 & Media & Media & Dudoso & Rz Seguro & Baja & Alta & Seguro & Dudoso & Media & Baja & Dudoso & Seguro \\
\hline 27 & 1 & 2017 & Media & Media & Rz Seguro & Rz Seguro & Baja & Baja & Rz Seguro & Seguro & Baja & Baja & Dudoso & Rz Seguro \\
\hline 28 & 1 & 2017 & Media & Media & Seguro & Rz Seguro & Baja & Baja & Rz Seguro & Rz Seguro & Media & Baja & Rz Seguro & Rz Seguro \\
\hline 29 & 1 & 2017 & Media & Media & Dudoso & Rz Seguro & Baja & Media & Dudoso & Rz Seguro & Baja & Alta & Dudoso & Rz Seguro \\
\hline 30 & 2 & 2017 & Media & Baja & Rz Seguro & Seguro & Muy Baja & Baja & Seguro & Rz Seguro & Baja & Baja & Rz Seguro & Rz Seguro \\
\hline 31 & 2 & 2017 & Media & Media & Rz Seguro & Seguro & Muy Baja & Baja & Seguro & Rz Seguro & Baja & Baja & Seguro & Rz Seguro \\
\hline 32 & 2 & 2017 & Alta & Media & Rz Seguro & Seguro & Baja & \begin{tabular}{|l|} 
Muy Baja \\
\end{tabular} & Seguro & Seguro & Baja & Baja & Rz Seguro & Seguro \\
\hline 33 & 2 & 2017 & Baja & Media & Rz Seguro & Seguro & Baja & Baja & Rz Seguro & Seguro & Baja & Baja & Seguro & Seguro \\
\hline 34 & 2 & 2017 & Media & Baja & Seguro & Seguro & Muy Baja & Muy Baja & Seguro & Seguro & Baja & Muy Baja & Rz Seguro & Seguro \\
\hline 35 & 2 & 2017 & Media & Media & Rz Seguro & Seguro & Muy Baja & Muy Baja & Seguro & Rz Seguro & Baja & Media & Seguro & Seguro \\
\hline
\end{tabular}

La recolección de datos se realizó en dos momentos distintos, donde cada equipo completó en cada instancia un formulario (ver Fig. 1), asignando valores a cada factor y calificando el valor asignado con un nivel de confianza. El formulario incluye los valores admisibles para cada factor y su reglas de interacción invalidante y limitante (de corresponder), anexado con una descripción de cada factor. La primera recolección tuvo lugar luego de que cada equipo realizó una o dos entrevistas abiertas para obtener una visión global del macrosistema en la organización correspondiente. Previamente, los equipos habían recibido instrucciones generales del proceso de IR a llevar a cabo. A medida que los equipos avanzaban en el proceso se fueron dando 
explicaciones detalladas de cada etapa del mismo. La otra recolección de datos se realizó al finalizar el proceso de IR, donde nuevamente se completó el mismo formulario, sin tener acceso a los valores iniciales asignados. Cabe mencionar que los equipos no tuvieron conocimiento sobre el propósito de la toma de datos solicitada. La Tabla 2 muestra una planilla parcial con datos crudos de 3 factores situacionales obtenidos en las dos tomas de datos realizadas en los 35 proyectos.

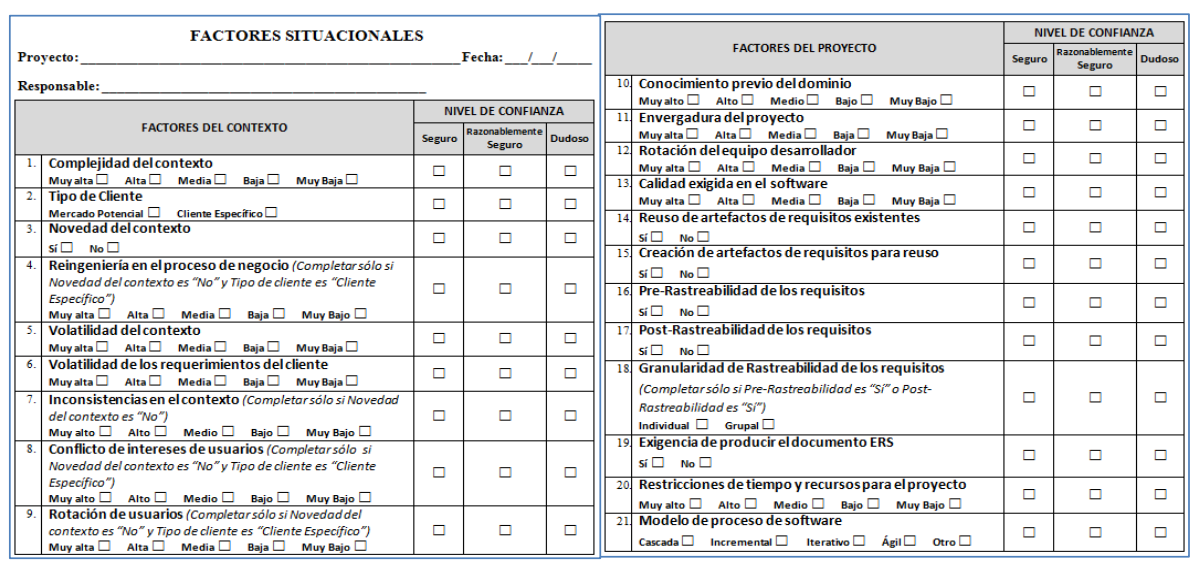

Fig. 1. Formulario de Recolección de Datos de Factores Situacionales (Fuente: [21])

\section{$5 \quad$ Análisis de Resultados}

En un pre-proceso de los datos adquiridos, para cada factor se descartaron aquellos proyectos con datos incompletos en el valor inicial, el valor final, el nivel de confianza inicial o el nivel de confianza final. Esto se puede apreciar en la Tabla 2 con aquellos datos tachados, donde por ejemplo, para el factor Volatilidad de los Requerimientos de los Clientes se descartaron 2 proyectos. Se considera que deberá realizarse un estudio que permita analizar una posible explicación a los proyectos con omisiones en la información. Obviamente, se han considerado especialmente aquellas situaciones en las que debido a dependencias entre factores, carecía de sentido asignarle valor a alguno de ellos. Esto se evidencia en la Tabla 3, donde los factores con un alto porcentaje de proyectos inválidos (Reingeniería en el Proceso de Negocio, Inconsistencias en el Contexto, Conflicto de Intereses de Usuarios, Rotación de Usuarios y Granularidad de Rastreabilidad de Requisitos) son claramente aquellos factores dependientes de otros por relaciones del tipo invalidantes.

Se utilizaron técnicas de la estadística descriptiva, para el análisis de la valoración de factores, habiéndose obtenido los totales por cada valor posible inicial y final, la cantidad de valores que cambiaron y la cantidad de valores que cambiaron a valores no próximos. Esto último significa que la valoración "Muy Alta" o "Alta" dada a un factor es considerada en el mismo rango de valores y, por lo tanto, no se los considera como un cambio relevante; lo mismo ocurre entre "Baja" y "Muy Baja". Esto no aplica a valores binarios (ejemplo: Tipo de Cliente) o valores nominales no ordinales 
(ejemplo: Modelo de Proceso de Software).

En base a los valores finales de los factores, estos se pueden resumir en los siguientes aspectos que caracterizaran los proyectos:

- El $66 \%$ de los proyectos fueron de complejidad media y el resto de complejidad baja a muy baja.

- El $49 \%$ de los proyectos eran de mediana envergadura y el otro $49 \%$ de pequeña envergadura, con un solo proyecto catalogado de gran envergadura.

- El $82 \%$ tuvieron una volatilidad del contexto entre baja y muy baja, solo 4 proyectos consideraron volatilidad alta o muy alta.

- El $76 \%$ de los proyectos eran con baja/muy baja inconsistencia del contexto.

- El $81 \%$ percibió una baja/muy baja rotación de usuarios.

- El 76\% de los proyectos tuvieron bajo/muy bajo conocimiento previo del dominio relativo a la organización, se presentó un solo proyecto con alto conocimiento.

- El 63\% indicó baja/muy baja rotación del equipo y los restantes un nivel medio.

- El 53\% percibió un nivel alto/muy alto en la calidad necesaria del software y un $44 \%$ calidad media, donde un solo proyecto calificó como baja la calidad exigida.

- El 64\% aplicaron baja/muy baja reingeniería en el proceso de negocio, con un solo proyecto que aplicó alta reingeniería; el resto aplicó una reingeniería media.

- El 76\% informó una baja/muy baja volatilidad en los requerimientos de los clientes, con un solo proyecto con alta volatilidad.

- El 89\% de los proyectos tuvieron un nivel bajo/muy bajo de conflicto de intereses, sin ningún proyecto con alta conflictividad.

- El $62 \%$ consideró que no se requería crear artefactos de requisitos para reuso, aunque el restante $38 \%$ estimó que sí.

- El 50\% consideró que las restricciones impuestas de tiempo (16 semanas) y recursos (cantidad de miembros del equipo) eran medias, mientras que de los restantes proyectos la mitad consideró que eran limitaciones altas y la otra mitad bajas.

Cada proyecto tenía un cliente específico dado que se desarrollaba para una organización real. Sin embargo, 4 proyectos inicialmente evaluaron el factor Tipo de Cliente incorrectamente, y posteriormente 3 de ellos lo rectificaron. Aunque todos los proyectos eran para contextos existentes, hubo un equipo que percibió incorrectamente que el desarrollo era para un contexto nuevo y lo calificó además como seguro.

Aunque en todos los proyectos se elaboró el Documento de Especificación de Requisitos, en 6 proyectos (18\%) no percibieron tal exigencia. Ningún proyecto hizo reuso de artefactos dado que no se disponía de un repositorio de artefactos de requisitos; sin embargo, el $20 \%$ de los proyectos calificó incorrectamente a este factor.

Los proyectos debían manejar Pre-rastreabilidad entre los artefactos construidos durante el proceso. El 15\% no percibió la realización de esta actividad como tal, aun cuando fue realizada. En la preparación no se dieron indicaciones sobre la Postrastreabilidad dado que era una tarea no involucrada en el proceso de IR, por lo que los resultados de este factor se descartaron para el análisis. Respecto a la Granularidad de Rastreabilidad de los Requisitos, esta se llevó a cabo en todos los proyectos en forma individual por requisito. Debe tenerse en cuenta que este factor depende de los otros dos factores relacionados con rastreabilidad (interacción invalidante), por lo que 
hubo solo 13 proyectos con valores iniciales y finales y 14 proyectos sin valor inicial por su dependencia de los otros dos factores, constituyéndose efectivamente en 27 proyectos considerados válidos, de los cuales el $48 \%$ percibió un manejo de la rastreabilidad individual.

Respecto al factor Modelo de Proceso de Software, el tema no fue considerado, pues solo se llevó a cabo el proceso de IR hasta obtener el conjunto más completo posible de requisitos, habiendo iteraciones entre las etapas, ya sea por correcciones o por mejor comprensión del macrosistema. Por tanto, se descartó el análisis de este factor, aunque por los resultados se observa que no fue bien comprendido.

Por otro lado, se hizo una evaluación del nivel de confianza con el que se calificó a los valores asignados a cada factor. Para ello, se calcularon el total de proyectos con apreciaciones seguras, razonablemente seguras y dudosas para los valores iniciales y finales de cada factor, junto con la cantidad de factores que mantuvieron su calificación de confianza (discriminando entre los que se mantuvieron en seguros o razonablemente seguros y los que se mantuvieron dudosos), los que mejoraron (por ejemplo: de dudoso a razonablemente seguro) y los que empeoraron (discriminando si empeoraron a razonablemente seguro o a dudoso). Estos datos se presentan en la Tabla 3, donde se observa que 2 factores invariantes tuvieron la mayor cantidad de proyectos que mantuvieron su nivel de confianza en seguro o razonablemente seguro: Tipo de Cliente y Novedad del Contexto, mientras que los 3 factores que en mayor medida mejoraron el nivel de confianza también son invariantes: Complejidad del Contexto, Creación de Artefactos para Reuso y Pre-Rastreabilidad de Requisitos.

Tabla 3. Resultados de Nivel de Confianza por Factor, siendo S: Seguro, RS: Razonablemente Seguro y D: Dudoso.

\begin{tabular}{|c|c|c|c|c|c|c|c|c|c|c|c|c|c|c|}
\hline \multirow[b]{3}{*}{ Factores Situacionales } & \multirow{3}{*}{\begin{tabular}{|c|}
$\begin{array}{c}\text { Proyectos } \\
\text { Incom- } \\
\text { pletos }\end{array}$ \\
\end{tabular}} & \multirow{3}{*}{$\begin{array}{c}\text { \% Proyectos } \\
\text { Incom- } \\
\text { pletos }\end{array}$} & \multirow{3}{*}{\begin{tabular}{|c|} 
Total \\
Proyectos \\
Válidos \\
\end{tabular}} & \multicolumn{11}{|c|}{ Nivel de Confianza } \\
\hline & & & & \multicolumn{3}{|c|}{ Inicial } & \multicolumn{3}{|c|}{ Final } & \multirow[b]{2}{*}{ Mejoró } & \multicolumn{2}{|c|}{ Se mantuvo en } & \multicolumn{2}{|c|}{ Empeoró a .... } \\
\hline & & & & $s$ & RS & D & $\mathrm{s}$ & RS & D & & S/RS & D & RS & D \\
\hline Complejidad del contexto & 0 & $0 \%$ & 35 & 13 & 19 & 3 & 27 & 8 & 0 & $54 \%$ & $37 \%$ & $0 \%$ & $9 \%$ & $0 \%$ \\
\hline Tipo de cliente & 0 & $0 \%$ & 35 & 26 & 7 & 2 & 29 & & 0 & $17 \%$ & $74 \%$ & $0 \%$ & $9 \%$ & $0 \%$ \\
\hline Novedad del contexto & 0 & $0 \%$ & 35 & 28 & 7 & 0 & 27 & 8 & 0 & $11 \%$ & $74 \%$ & $0 \%$ & $14 \%$ & $0 \%$ \\
\hline Reingeniería en el proceso de negocio & 7 & $20 \%$ & 28 & 11 & 17 & 0 & 20 & 8 & 0 & $43 \%$ & $46 \%$ & $0 \%$ & $11 \%$ & $0 \%$ \\
\hline \begin{tabular}{|l|} 
Volatilidad del contexto \\
\end{tabular} & 1 & $3 \%$ & 34 & 21 & 11 & & 20 & 13 & & $26 \%$ & $50 \%$ & $0 \%$ & $21 \%$ & $3 \%$ \\
\hline Volatilidad de los requerimiento & 2 & $6 \%$ & 33 & 15 & 14 & 4 & 23 & 8 & 2 & $42 \%$ & $45 \%$ & $0 \%$ & $6 \%$ & $6 \%$ \\
\hline Inconsistencias en el contexto & 6 & $17 \%$ & 29 & 11 & 13 & 5 & 18 & 8 & 3 & $45 \%$ & $41 \%$ & $0 \%$ & $3 \%$ & $10 \%$ \\
\hline Conflicto de intereses de usuari & 8 & $23 \%$ & 27 & 16 & 8 & 2 & 23 & & & $33 \%$ & $59 \%$ & $0 \%$ & $4 \%$ & $4 \%$ \\
\hline Rotación de usuarios & 9 & $26 \%$ & 26 & 18 & 6 & 2 & 19 & 6 & 1 & $23 \%$ & $58 \%$ & $0 \%$ & $15 \%$ & $4 \%$ \\
\hline Conocimiento previo del dominio & 1 & $3 \%$ & 34 & 24 & 10 & 0 & 26 & 6 & 2 & $24 \%$ & $53 \%$ & $0 \%$ & $18 \%$ & $6 \%$ \\
\hline Envergadura del proyecto & 0 & $0 \%$ & 35 & 13 & 19 & 3 & 22 & 10 & 3 & $40 \%$ & $37 \%$ & $0 \%$ & $14 \%$ & $9 \%$ \\
\hline Rotación del equipo desarrollad & 0 & $0 \%$ & 35 & 18 & 10 & 7 & 19 & 13 & 3 & $34 \%$ & $37 \%$ & $6 \%$ & $20 \%$ & $3 \%$ \\
\hline \begin{tabular}{|l|} 
Calidad exigida en el software \\
\end{tabular} & 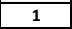 & 70 & 34 & 14 & 16 & 4 & 22 & 10 & 2 & $35 \%$ & $53 \%$ & $6 \%$ & $6 \%$ & $0 \%$ \\
\hline Reuso de artefactos de requisitos existentes & 0 & $0 \%$ & 35 & 21 & 6 & 8 & 28 & 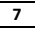 & 0 & $37 \%$ & $51 \%$ & $0 \%$ & $11 \%$ & $0 \%$ \\
\hline Creación de artefactos de requisitos para reuso & 1 & $3 \%$ & 34 & 13 & 16 & 5 & 23 & 9 & 2 & $50 \%$ & $32 \%$ & $0 \%$ & $12 \%$ & $6 \%$ \\
\hline Pre-Rastreabilidad de los re & 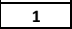 & $3 \%$ & 34 & 10 & 15 & 9 & 20 & 12 & 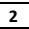 & $50 \%$ & $32 \%$ & $6 \%$ & $12 \%$ & $0 \%$ \\
\hline Post-Rastreabilidad c & 1 & $3 \%$ & 34 & 12 & 13 & 9 & 16 & 14 & 4 & $41 \%$ & $32 \%$ & $9 \%$ & $15 \%$ & $3 \%$ \\
\hline Granularidad de rastreabilidad de requisitos & 22 & $63 \%$ & 13 & 3 & 6 & 4 & 6 & 6 & 1 & $46 \%$ & $38 \%$ & $8 \%$ & $8 \%$ & $0 \%$ \\
\hline Exigencia de producir el documento EF & 2 & $6 \%$ & 33 & 18 & 10 & 5 & 28 & 4 & 1 & $42 \%$ & $52 \%$ & $0 \%$ & $3 \%$ & $3 \%$ \\
\hline Restriccio & 1 & $3 \%$ & 34 & 13 & 16 & 5 & 17 & 16 & 1 & $35 \%$ & $44 \%$ & $3 \%$ & $18 \%$ & $0 \%$ \\
\hline Modelo de proceso de software & 1 & $3 \%$ & 34 & 11 & 19 & 4 & 22 & 11 & 1 & $44 \%$ & $44 \%$ & $3 \%$ & $9 \%$ & $0 \%$ \\
\hline
\end{tabular}

Asimismo, se estudiaron los cambios de nivel de confianza sobre aquellos valores que sufrieron cambios relevantes (ver Tabla 4). En otras palabras, por factor se calcularon las cantidades de proyectos cuya valoración inicial fue percibida dudosa, razonablemente segura o segura, para los proyectos con cambios a valores no próximos. 
Tabla 4. Resultados de la Evolución de Valores y de Nivel de Confianza

\begin{tabular}{|c|c|c|c|c|c|c|c|c|c|}
\hline \multirow[b]{2}{*}{ Factores Situacionales } & \multirow[b]{2}{*}{\begin{tabular}{|c|} 
Total de \\
Proyectos \\
Válidos
\end{tabular}} & \multirow[b]{2}{*}{$\begin{array}{c}\% \text { Nivel de } \\
\text { Confianza } \\
\text { modificado }\end{array}$} & \multirow[b]{2}{*}{\begin{tabular}{|c|}
$\%$ \\
Dudosos \\
al inicio
\end{tabular}} & \multirow[b]{2}{*}{$\begin{array}{c}\% \\
\text { Dudosos } \\
\text { al final }\end{array}$} & \multicolumn{5}{|c|}{ Análisis sobre Proyectos con Cambios Relevantes } \\
\hline & & & & & $\begin{array}{c}\text { Proyectos } \\
\text { con } \\
\text { Cambios } \\
\text { Relevantes }\end{array}$ & $\begin{array}{c}\text { \% Valores } \\
\text { con Cambios } \\
\text { Relevantes }\end{array}$ & $\left|\begin{array}{c}\% \text { Dudosos } \\
\text { al inicio }\end{array}\right|$ & $\begin{array}{c}\text { \% Seguros } \\
\text { al inicio }\end{array}$ & $\begin{array}{c}\text { \% Raz. } \\
\text { Seguros al } \\
\text { inicio }\end{array}$ \\
\hline Complejidad del contexto & 35 & $63 \%$ & $9 \%$ & $0 \%$ & 13 & $37 \%$ & $0 \%$ & $38 \%$ & $62 \%$ \\
\hline Tipo de cliente & 35 & $26 \%$ & $6 \%$ & $0 \%$ & 3 & 9\% & $67 \%$ & $0 \%$ & $33 \%$ \\
\hline Novedad del contexto & 35 & $26 \%$ & $0 \%$ & $0 \%$ & 4 & $11 \%$ & $0 \%$ & $50 \%$ & $50 \%$ \\
\hline Reingeniería en el proceso de negocio & 28 & $54 \%$ & $0 \%$ & $0 \%$ & 15 & $54 \%$ & $0 \%$ & $33 \%$ & $67 \%$ \\
\hline Volatilidad del contexto & 34 & $50 \%$ & $6 \%$ & $3 \%$ & 6 & $18 \%$ & $17 \%$ & $33 \%$ & $50 \%$ \\
\hline Volatilidad de los requerimientos del cliente & 33 & $55 \%$ & $12 \%$ & $6 \%$ & 11 & $33 \%$ & $18 \%$ & $27 \%$ & $55 \%$ \\
\hline Inconsistencias en el contexto & 29 & $59 \%$ & $17 \%$ & $10 \%$ & 8 & $28 \%$ & $13 \%$ & $50 \%$ & $38 \%$ \\
\hline Conflicto de intereses de usuarios & 27 & $41 \%$ & $7 \%$ & $4 \%$ & 4 & $15 \%$ & $25 \%$ & $25 \%$ & $50 \%$ \\
\hline Rotación de usuarios & 26 & $42 \%$ & $8 \%$ & $4 \%$ & 7 & $27 \%$ & $14 \%$ & $71 \%$ & $14 \%$ \\
\hline Conocimiento previo del dominio & 34 & $47 \%$ & $0 \%$ & $6 \%$ & 12 & $35 \%$ & $0 \%$ & $67 \%$ & $33 \%$ \\
\hline Envergadura del proyecto & 35 & $63 \%$ & $9 \%$ & $9 \%$ & 16 & $46 \%$ & $6 \%$ & $38 \%$ & $56 \%$ \\
\hline Rotación del equipo desarrollador & 35 & $57 \%$ & $20 \%$ & $9 \%$ & 14 & $40 \%$ & $14 \%$ & $64 \%$ & $21 \%$ \\
\hline Calidad exigida en el software & 34 & $41 \%$ & $12 \%$ & $6 \%$ & 18 & $53 \%$ & $17 \%$ & $28 \%$ & $56 \%$ \\
\hline Reuso de artefactos de requisitos existentes & 35 & $49 \%$ & $23 \%$ & $0 \%$ & 12 & $34 \%$ & $33 \%$ & $50 \%$ & $17 \%$ \\
\hline Creación de artefactos de requisitos para reuso & 34 & $68 \%$ & $15 \%$ & $6 \%$ & 14 & $41 \%$ & $21 \%$ & $43 \%$ & $36 \%$ \\
\hline Pre-Rastreabilidad de los requisitos & 34 & $62 \%$ & $26 \%$ & $6 \%$ & 15 & $44 \%$ & $20 \%$ & $33 \%$ & $47 \%$ \\
\hline Post-Rastreabilidad de los requisitos & 34 & $59 \%$ & $26 \%$ & $12 \%$ & 12 & $35 \%$ & $17 \%$ & $33 \%$ & $50 \%$ \\
\hline Granularidad de rastreabilidad de requisitos & 13 & $54 \%$ & $31 \%$ & $8 \%$ & 3 & $23 \%$ & $33 \%$ & $33 \%$ & $33 \%$ \\
\hline Exigencia de producir el documento ERS & 33 & $48 \%$ & $15 \%$ & $3 \%$ & 10 & $30 \%$ & $20 \%$ & $60 \%$ & $20 \%$ \\
\hline Restricciones de tiempo y recursos del proyecto & 34 & $53 \%$ & $15 \%$ & $3 \%$ & 13 & $38 \%$ & $8 \%$ & $46 \%$ & $46 \%$ \\
\hline Modelo de proceso de software & 34 & $53 \%$ & $12 \%$ & $3 \%$ & 13 & $38 \%$ & $8 \%$ & $23 \%$ & $69 \%$ \\
\hline
\end{tabular}

Hubo 13 factores situacionales con más de un $50 \%$ de proyectos que modificaron su nivel de confianza (ver columna "\% Nivel de Confianza Modificado" en Tabla 4), siendo esto independiente del tipo de evolución del factor, pues 6 de esos factores eran invariantes y para los que se presumía una mejor apreciación inicial. Esto evidencia la dificultad en establecer valores confiables al inicio del proceso de IR. Por otro lado, hubo algunos factores, en una escasa cantidad de proyectos, cuya valoración final fue dudosa, los que en su mayoría tenían números altos en cantidad de cambios en el nivel de confianza, tales como: Inconsistencias en el Contexto y Envergadura del Proyecto.

En cuanto a cambios relevantes en la valoración de los factores (ver columnas en color en Tabla 4), hubo 2 factores donde más del 50\% de los proyectos cambiaron su valor inicial: Reingeniería en el Proceso de Negocio y Calidad Exigida del Software, siendo el primero un factor contingente y el segundo invariante. Para el primer factor, esto podría implicar la necesidad de prestarle una mayor atención al momento de valorarlo, mientras que para el segundo factor es posible que su significado fuese ambiguo o no fuera clara la manera de lograr un conocimiento sobre el mismo. Otros 2 factores invariantes tuvieron también porcentajes altos de cambio, donde la Envergadura del Proyecto puede asociarse a la misma problemática del factor Reingeniería en el Proceso de Negocio, y la Pre-Rastreabilidad de Requisitos puede tener la misma dificultad de valoración que Calidad Exigida del Software. En estos 4 factores el nivel de confianza dado inicialmente a los valores que luego cambiaron fue "Razonablemente Seguro" en un porcentaje muy elevado.

Además, se corrobora que los factores con mayor porcentaje de nivel de confianza inicial "Seguro" respecto a cambios relevantes en sus valores, tuvieron efectivamente baja cantidad de cambios relevantes (ver en Tabla 4), tal es el caso de Rotación de 
Usuarios y Conocimiento Previo del Dominio.

Análisis del Rigor de la Aplicación del Estudio de Caso. El fenómeno bajo estudio consistió en un conjunto de factores situacionales y la calidad con que se los estima en el transcurso de un proceso de requisitos desarrollado en organizaciones reales, con el propósito de describir y en lo posible explicar esas calidades y evoluciones, para poder luego refinar una estrategia de adaptación dinámica del proceso de IR. Es decir, se estudió el fenómeno en su contexto natural. Este estudio se basó en múltiples fuentes de evidencia: 35 proyectos, cuyos datos se analizaron transversalmente para cada factor, logrando una razonable confiabilidad. La descripción presentada de los pasos seguidos y bajo qué condiciones se realizó la recolección de datos permitirá la realización de replicaciones, contribuyendo a la confiabilidad experimental. Respecto a la validez de construcción, los datos recolectados se han mostrado parcialmente en la Tabla 2, basado en la toma de datos a través de formularios estandarizados para todos los proyectos. Respecto a la validez interna, el análisis de los datos se presentan en las Tablas 3 y 4, donde se describe la evolución de los valores de los factores y del nivel de confianza de los mismos en porcentajes de proyectos considerados. Los equipos fueron homogéneos, recibiendo el mismo nivel de capacitación para llevar a cabo su proyecto. Respecto a la validez externa, se planeó el conjunto de factores a estudiar en base a trabajos propios [22] y de otros autores en IMS y Variabilidad de Procesos. Además, debe considerarse que se puso especial cuidado en la elección de los proyectos involucrados. Este estudio de caso ha permitido tener una mejor comprensión de la evolución de los factores y su interrelación, y de los riesgos de cometer errores en su estimación y qué factores son más sensibles a esos riesgos. Esto permitirá realizar mejoras a las guías de adaptación del proceso de IR en dos aspectos: en cuanto a la heurística de evaluación y análisis de los factores situacionales en cada punto de variación y en cuanto a la descripción específica de cada factor.

\section{Conclusiones y Futuros Trabajos}

La adaptación situacional de los procesos depende fuertemente de la calidad de los factores situacionales, pues la eliminación, reemplazo o adición de una actividad es función directa de los valores que se le asignan a dichos factores. Esa asignación es en realidad una estimación que debe ser lo más aproximada posible a la situación efectivamente imperante. Por otro lado, dependiendo de la naturaleza del proceso y de la adaptación que se trate, la eliminación, reemplazo o adición innecesaria de una actividad puede tener una importancia diferente, sin embargo, es claro que en condiciones similares, la primera de ellas es mucho más perniciosa que las otras dos. Además, esta adaptación puede verse comprometida cuando estos factores evolucionan y ello no es percibido oportunamente. Una valoración imprecisa es perniciosa y la evolución de la situación puede agravar la calidad de esa valoración. A pesar de la variedad de estudios relacionados con la IMS, estos aspectos no son tratados con la profundidad necesaria, lo que resulta en una carencia grave.

A partir de este estudio de caso se ha podido observar una alta variabilidad en la 
estimación de los factores situacionales. Se han identificado factores que sufrieron cambios relevantes en más del $40 \%$ de los proyectos. Además, la gran mayoría de los factores (exceptuando solo 2) tuvo arriba del $40 \%$ de los proyectos con cambios en el nivel de confianza de la estimación del factor. Estos resultados no pueden generalizarse; solo describen cuestiones no evidentes, aunque acotadas a un conjunto de proyectos de pequeña a mediana envergadura, de una complejidad baja a media y desarrollados por equipos con poca experiencia en IR.

En resumen, este estudio permitió establecer con evidencia observable aquellos factores situacionales que cambian más frecuentemente o son mal estimados en el proceso específico de IR puesto en práctica. Como consecuencia, se les debe prestar mayor atención antes y durante la definición y re-adaptación del proceso, teniendo en consideración que la valoración de algunos factores además impacta en la valoración de otros y, por ende, puede desencadenar otro tipo de ajustes en el proceso.

Del análisis de los resultados del estudio de caso presentado, se observa la necesidad de disponer de un proceso de requisitos que se ajuste dinámicamente durante su desarrollo. Gran parte de estas adaptaciones a lo largo del proceso se deben principalmente a cambios por apreciaciones imprecisas de la situación. Se ha podido detectar que las valoraciones inciertas o erróneas de estos factores se deben en gran parte a la falta de comprensión del contexto y, en menor medida, al desconocimiento de algunas actividades de la IR o dificultad de interpretación de las definiciones de algunos factores. Respecto a la primera causa, se propone definir una heurística que ayude a una valoración temprana menos dudosa y/o que mida las consecuencias de una adaptación imprecisa y permita redefinir transitoriamente esos valores iniciales para una adaptación del proceso que mitigue futuros cambios. Para una mejor comprensión del significado de los factores situacionales, se mejorará la definición de los mismos, tratando de reducir ambigüedades y ofreciendo precisiones sobre sus posibles valores.

\section{Referencias}

1. Carrizo, D., Dieste, O., Juristo, N.: Study of elicitation techniques adequacy. En: 11th Workshop on Requirements Engineering, pp. 104-114. Barcelona, España (2008).

2. Wheelen, T.L., Hunger, J.D.: Strategic Management and Business Policy: Toward Global Sustainability, $13^{\circ}$ ed., Pearson (2012).

3. Cetina, C., Giner, P., Fons, J., Pelechano, V.: Autonomic Computing Through Reuse of Variability Models at Runtime: The Case of Smart Homes. Computer, IEEE Computer Society, 42(10), 37-43 (2009).

4. Simidchieva, B., Clarke, L., Osterweil, L.: Representing Process Variation with a Process Family. Software Process Dynamics and Agility. ICSP 2007. Lecture Notes in Computer Science, vol. 4470, pp. 109-120. Springer, Berlín (2007).

5. Santos, E., Castro, J., Sánchez, J., Pastor, O.: A Goal-Oriented Approach for Variability in BPMN. 13th Workshop on Requirements Engineering, pp.17-28. Cuenca, Ecuador (2010).

6. Hallerbach, A., Bauer, T., Reichert, M.: Managing Process Variants in the Process Lifecycle. 10th Intl Conference on Enterprise Information Systems, pp.154-161. España (2008).

7. Galster, M., Weyns, D., Tofan, D., Michalik, B., Avgeriou, P.: Variability in Software Systems - A Systematic Literature Review. IEEE Transactions on Software Engineering, 40(3), 282-306 (2014). 
8. Brinkkemper, S.: Method Engineering: Engineering of Information Systems Development Methods and Tools. Information and Software Technology, 38(4), 275-280 (1996).

9. Rolland, C.: Method Engineering: Towards Methods as Services. Making Globally Distributed Software Development a Success Story. En: Intl Conference on Software Process (ICSP 2008), pp.10-11. Leipzig, Alemania (2008).

10. Bucher, T., Klesse, M., Kurpjuweit, S., Winter, R.: Situational Method Engineering. Situational method engineering: fundamentals and experiences, Springer US, pp. 33-48 (2007).

11. Khan, H.H., bin Mahrin, M., bt Chuprat, S.: Factors for Tailoring Requirement Engineering Process: A Review. Intl Journal of Software Engineering and Technology, 1(1), 7-18 (2014).

12. Henderson-Sellers, B., Ralyté, J.: Situational Method Engineering: State-of-the-Art Review. Journal of Universal Computer Science, 16(3), 424-478 (2010).

13. Zowghi, D., Firesmith, D., Henderson-Sellers, B.: Using the OPEN Process Framework to Produce a Situation-Specific Requirements Engineering Method. En: 1st Intl Workshop on Situational Requirements Engineering Processes, pp.59-74. París, Francia (2005).

14. Jiang, L., Eberlein, A.: A Framework for Requirements Engineering Process Development (FRERE). 19th Australian Conference on Software Engineering. Perth, Australia (2008).

15. Hickey, A.M., Davis, A.M.: A Unified Model of Requirements Elicitation. Journal of Management Information Systems, 20(4), 65-84 (2004).

16. Hickey A.M., Davis A.M.: An Ontological Approach to Requirements Elicitation Technique Selection. En: Sharman R., Kishore R., Ramesh R. (eds) Ontologies. Integrated Series in Information Systems, vol. 14. Springer, Boston, MA (2007).

17. Bakhat, K.A., Sarwar, A.A., Motla, Y.H.B., Akhtar, M.C.: A Situational Requirement Engineering Model for an Agile Process. Bahria University Journal of Information \& Communication Technology, 8(1), 21-26 (2015).

18. Khan, H., bin Mahrin, M., Mali, M.: Situational Requirement Engineering Framework for Global Software Development: Formulation and Design. Bahria University Journal of Information \& Communication Technologies, 9(1), 74-84 (2016).

19. Coulin, C., Zowghi, D., Sahraoui, A.: A Situational Method Engineering Approach to Requirements Elicitation Workshops in the Software Development Process. Software Process: Improvement and Practice, 11(5), 451-464 (2006).

20. Jafarinezhad, O., Ramsin, R.: Development of Situational Requirements Engineering Processes: A Process Factory Approach. En: 36th IEEE Intl Conference on Computer Software and Applications, pp. 279-288 (2012).

21. Ledesma, V.A., Hadad, G.D.S., Doorn, J.H.: Adaptación Dinámica de un Proceso de Requisitos Orientado al Cliente. En: 4to Congreso Nacional de Ingeniería Informática / Sistemas de Información. Salta, Argentina. ISSN: 2347-0372 (2016).

22. Hadad, G.D.S., Ledesma, V.A., Doorn, J.H.: Proceso de Requisitos Adaptable a Factores Situacionales. En: XVI Workshop de Investigadores en Ciencias de la Computación, pp. 448-452. Ushuaia, Argentina (2014).

23. Leite, J.C.S.P., Doorn, J.H., Kaplan, G.N., Hadad, G.D.S., Ridao, M.: Defining System Context using Scenarios. Perspectives on Software Requirements, Kluwer Academic Publishers, cap.8, pp.169-199 (2004).

24. Kyburz-Graber, R.: Does case-study methodology lack rigour? The need for quality criteria for sound case-study research, as illustrated by a recent case in secondary and higher education. Journal of Environmental Education Research, 10(1), 53-65 (2004).

25. Yin, R. K.: The case study crisis: some answers. Administrative Science Quarterly, 26(1), 58-65 (1981). 